\title{
Wave and Material Properties of Marine Sediments: Theoretical Relationships for Geoacoustic Inversions
}

\author{
Michael J. Buckingham \\ Marine Physical Laboratory, Scripps Institution of Oceanography \\ University of California, San Diego, 9500 Gilman Drive, La Jolla, CA 92093-0238, USA \\ Also affiliated to: Institute of Sound and Vibration Research \\ The University, Southampton SO17 1BJ, UK
}

\begin{abstract}
In recent years, a theory of wave propagation in marine sediments has been developed, based on the grain-to-grain interactions that occur during the passage of compressional and shear waves. The theory yields a dispersion pair, representing phase speed and attenuation, for each wave. These expressions are functions of frequency and the physical properties of the sediment, that is, the porosity, density, grain size and over-burden pressure (or depth in the medium). The predicted functional dependencies are compared with extensive data sets that have appeared in the literature over the past couple of decades. No adjustable parameters are available to help improve the comparisons. In all cases, the theory shows a high level of agreement with the data. This agreement even extends to both attenuations, in that the theory, which predicts intrinsic attenuation, arising from the conversion of wave energy into heat, accurately traces out the lower bound of the widelydistributed measurements. This is physically reasonable, since the data represent effective attenuation, which includes additional sources of loss such as scattering from shell fragments and other inhomogeneities in the medium. It is suggested that the set of simple algebraic expressions comprising the theory have application in evaluating the geoacoustic parameters of the seabed, all of which may be computed from knowledge of just one, say the compressional wave speed or the porosity.
\end{abstract}

\section{INTRODUCTION}

Marine sediments are granular materials (sands, silts and clays), saturated with seawater, that support the propagation of a compressional (longitudinal) wave and a shear (transverse) wave. Commonly, sediments are unconsolidated, that is, the grains are unbonded with the facility to move relative to one another. The compressional wave of the second kind, or slow wave, predicted by Biot's theory $[1,2]$ of wave propagation in porous media has not been observed in unconsolidated sediments, despite several attempts to detect it. Either the slow wave is negligible in or absent from such materials and is not further considered here.

The wave properties of sediments are the phase speeds and attenuations of the compressional and shear waves. Over several decades, these wave properties have been carefully measured, both in situ and in core samples, by several groups of investigators. It is now well-established that the wave properties depend more or less systematically on 
the porosity, density and grain size of the medium, along with the measurement frequency and over-burden pressure, which translates into depth in the sediment. The correlations that have been observed to exist between the wave and physical properties of the medium have been expressed by Hamilton et al. [3,4] and Richardson and Briggs [5] through empirical regression equations.

An alternative approach to the correlations between the wave and physical properties of sediments has been pursued by Buckingham [6], who developed a theoretical model of wave propagation in saturated granular materials. The basis of his model is a particular form of inter-granular shearing, which yields dispersion relationships for the compressional and the shear wave. These dispersion relationships, in the form of algebraic expressions for the phase speed and the attenuation of both types of wave, depend explicitly on the grain size, porosity, depth in the sediment, and measurement frequency. They are independent of such parameters as the pore-fluid viscosity, the permeability and tortuosity, all of which are present in Biot's dispersion relationships $[1,2]$. Moreover, Buckingham's theory, unlike Biot's, does not include a bulk or shear frame modulus, since the stiffness of the material (i.e., the elasticity of the mineral "frame") is naturally accounted for by the grain-to-grain interactions.

In this paper, a brief comparison is made between Buckingham's theoretical dispersion relationships and data sets, taken from the literature, on the inter-relationships between the wave and physical properties of marine sediments. As will be demonstrated, the theoretical predictions accurately match the data in practically all regards.

\section{DISPERSION RELATIONS}

The expressions for the sound speed, $c_{p}$, and attenuation, $\alpha_{p}$, from Buckingham's intergranular shearing theory are as follows:

$$
c_{p}=\frac{c_{o}}{\operatorname{Re}\left[1+\frac{\gamma_{p}+(4 / 3) \gamma_{s}}{\rho_{o} c_{o}^{2}}(j \omega T)^{n}\right]^{-1 / 2}}
$$

and

$$
\alpha_{p}=-\frac{\omega}{c_{o}} \operatorname{Im}\left[1+\frac{\gamma_{p}+(4 / 3) \gamma_{s}}{\rho_{o} c_{o}^{2}}(j \omega T)^{n}\right]^{-1 / 2}
$$

The corresponding expressions for the shear wave speed, $c_{s}$, and attenuation, $\alpha_{s}$, are

$$
c_{s}=\sqrt{\frac{\gamma_{s}}{\rho_{o}}} \frac{(\omega T)^{n / 2}}{\cos \left(\frac{n \pi}{4}\right)}
$$

and 


$$
\alpha_{s}=\omega \sqrt{\frac{\rho_{o}}{\gamma_{s}}}(\omega T)^{-n / 2} \sin \left(\frac{n \pi}{4}\right)
$$

In these dispersion pairs, $j=\sqrt{-1}, \omega$ is angular frequency, $T$ is an arbitrary time, set equal to one second, and introduced to avoid awkward dimensions arising when frequency is raised to a fractional power, $\rho_{o}$ is the bulk density of the material, and $c_{o}$ is Wood's [7] sound speed in the equivalent suspension:

$$
c_{o}=\sqrt{\frac{\kappa_{o}}{\rho_{o}}},
$$

where $\rho_{o}$ is the bulk modulus of the medium.

Both $\rho_{o}$ and $\kappa_{o}$ may be expressed as weighted means of the respective values for the two constituent materials, the mineral grains $\left(\rho_{g}, \kappa_{g}\right)$ and pore fluid $\left(\rho_{w}, \kappa_{w}\right)$ :

$$
\rho_{o}=N \rho_{w}+(1-N) \rho_{g}
$$

and

$$
\frac{1}{\kappa_{o}}=N \frac{1}{\kappa_{w}}+(1-N) \frac{1}{\kappa_{g}},
$$

where $N$ is the fractional porosity. Representative values of the properties of the mineral grains and pore fluid are, respectively: $\rho_{g}=2730 \mathrm{~kg} / \mathrm{m}^{3}, \kappa_{g}=3.36 \times 10^{10} \mathrm{~Pa}$; and $\rho_{w}=$ $1005 \mathrm{~kg} / \mathrm{m}^{3}, \kappa_{w}=2.37 \times 10^{9} \mathrm{~Pa}$.

The three remaining parameters in the dispersion relationships, $\left(\gamma_{p}, \gamma_{s}, n\right)$, characterize grain-to-grain shearing that occurs during the passage of a wave. Analogous to the Lamé parameters of elasticity theory, the first two, $\gamma_{p}$ and $\gamma_{s}$, are (real) compressional and shear moduli, whereas the third, $n$, is a positive fractional index, which is a measure of the strain hardening that is postulated to occur at grain contacts as inter-granular sliding progresses. From the Hertz theory of elastic spheres in contact [8], the two moduli are expressed in terms of the porosity, $N$, grain diameter, $u_{g}$, and depth in the sediment, $d$, as follows:

$$
\gamma_{p}=\gamma_{p o}\left[\frac{(1-N) u_{g} d}{\left(1-N_{o}\right) u_{g o} d_{o}}\right]^{1 / 3}
$$

and 


$$
\gamma_{s}=\gamma_{s o}\left[\frac{(1-N) u_{g} d}{\left(1-N_{o}\right) u_{g o} d_{o}}\right]^{2 / 3}
$$

where the compressional and shear coefficients, $\gamma_{p o}$ and $\gamma_{s o}$, respectively, take numerical values that are independent of the bulk physical properties of the medium. Although $\left(\gamma_{p o}\right.$, $\left.\gamma_{s o}, n\right)$ may vary weakly from one sediment to another, due to microscopic differences between grain-surface properties, their values are held constant throughout the remaining discussion: $\gamma_{p o}=3.9 \times 10^{8} \mathrm{~Pa}, \gamma_{s o}=4.65 \times 10^{7} \mathrm{~Pa}$ and $n=0.0851$. (See Buckingham [9] for the derivation of these values). The three reference parameters, porosity $N_{o}$, grain size $u_{g_{o}}$, and depth $d_{o}$, in Eqs. (6), serve to avoid awkward dimensions appearing when the terms in square brackets are raised to a fractional power. These reference parameters are chosen for convenience $\left(N_{o}=0.37, u_{g o}=1000 \mu \mathrm{m}, d_{o}=0.3 \mathrm{~m}\right)$ and do not represent additional unknowns.

Equations (1) to (6) specify completely the predicted properties of the compressional and shear wave in a marine sediment. These expressions depend explicitly on grain size and porosity, and when measured values for both are available, they should be used in evaluating the theory. This, however, is not always possible. For instance, if the wave properties were to be evaluated as functions of porosity or of grain size, bearing in mind that the latter two are correlated, then it is necessary to identify a relationship connecting $N$ and $u_{g}$. From a random-packing, rough-grain argument, Buckingham [10] has derived such a relationship:

$$
N=1-P_{s}\left\{\frac{u_{g}+2 \Delta}{u_{g}+4 \Delta}\right\}^{3},
$$

where $P_{s}=0.63$ is the packing factor of a random "close" packing of uniform spheres and the rms roughness, or shape, parameter, $\Delta$, characterizes the degree of non-sphericity exhibited by the mineral grains in the sediment. It is possible that $\Delta$ may differ slightly from one sediment to another, but for the purpose of the following discussions it is held constant with the value $\Delta=1 \mu \mathrm{m}$.

The predicted wave properties as functions of frequency, porosity, grain size, and depth in the sediment, may now be investigated, using Eqs. (1) to (7) with the parameter values that have been cited. For the dispersion relations in Eqs. (1) and (2) to be valid, these theoretical functional dependencies must follow the trends of the corresponding data sets. Note that no adjustable parameters are available to help improve any of the following comparisons.

\section{FREQUENCY DEPENDENCE}

Straightforward approximations [6] of the dispersion relationships in Eqs. (1) and (2) indicate that both wave speeds show near-logarithmic dispersion and both attenuations 
vary almost linearly with the frequency. Recent measurements of the compressional wave properties in sand sediments by Simpson et al. [11,12], made at frequencies between 3 and $100 \mathrm{kHz}$, are consistent with the theory, indicating weak logarithmic dispersion and an attenuation that varies linearly with frequency.

Similar behavior is displayed by compressional-wave data discussed by Buckingham and Richardson [13] from the SAX99 medium sand sediment in the Gulf of Mexico. Fig. 1 shows the SAX99 measurements along with the corresponding theoretical curves from Eqs. (1). Both theory and experiment show logarithmic dispersion, at a level of approximately $1 \%$ per decade, whilst the measured and predicted attenuations exhibit a near linear dependence on frequency.

Few data sets are available on the shear wave speed and attenuation as functions of frequency. Brunson and Johnson [14] made laboratory measurements of shear attenuation in a medium sand and found a near-linear dependence on frequency over the frequency range from 0.45 to $7 \mathrm{kHz}$, consistent with Eq. (2b). Their attenuation data are reproduced in Buckingham's [6] Fig. 2a.
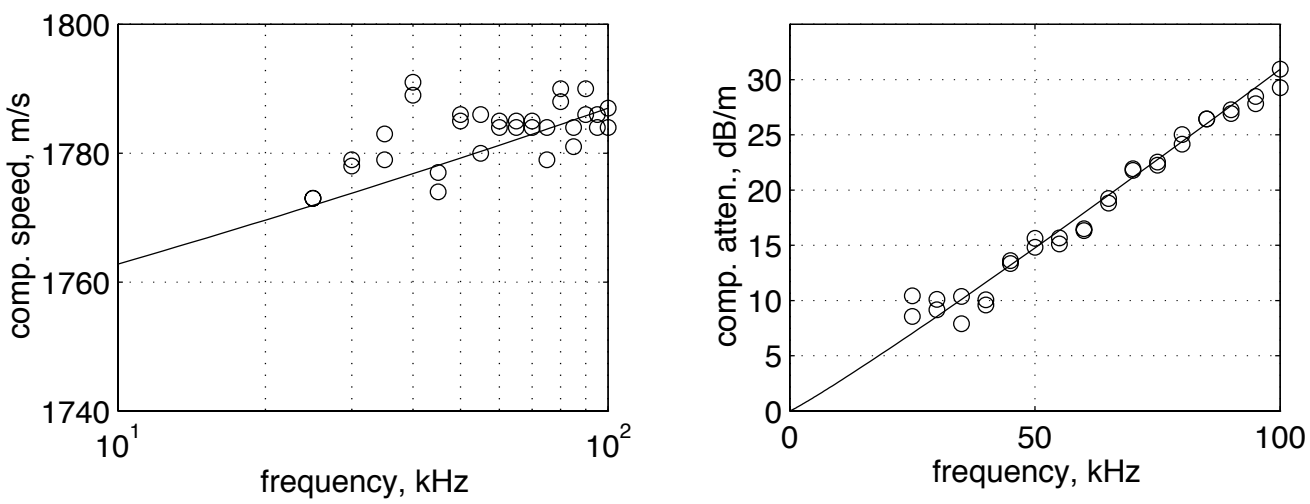

FIGURE 1. Sound speed and attenuation as functions of frequency. Note the semi-logarithmic and linear axes, respectively. Data are for the medium sand sediment at the SAX99 site.

\section{DEPTH DEPENDENCE}

Richardson et al. [15] have reported $400 \mathrm{kHz}$ measurements of the compressional wave speed and attenuation as functions of depth in diver-collected core samples. Figures $2 \mathrm{a}$ and $2 \mathrm{~b}$ show these data sets and for comparison the curves from Eqs. (1). The compressional speed ratio in Fig. 2a is the sound speed in the sediment normalized to that in seawater at the same temperature.

Below a depth of $10 \mathrm{~cm}$, the predicted wave speed falls within the limits of the data in Fig. 2a, but slightly under-estimates the measurements at shallower depths. This small discrepancy may be due to ducting caused by the steep gradient in the sound speed profile near the interface, which could lead to the measurements being over-estimated.

The theoretical attenuation profile in Fig. $2 b$ does not fall within the range of the widely distributed measurements but instead traces the lower limit of the data points. Such behavior is exactly as expected. The theory predicts the intrinsic attenuation, arising from the conversion of wave energy into heat. The data, on the other hand, represent the effective attenuation, which includes the intrinsic attenuation plus any additional sources of loss, due, for example, to scattering from inhomogeneities such as shell fragments that 
may be present in the medium. Thus the intrinsic attenuation should delineate the lower bound to the effective attenuation, as it does in Fig. $2 \mathrm{~b}$.

Shear wave speed and attenuation profiles have been reported by Richardson $[16,17]$ for a sand sediment in the North Sea, at a site designated C1. Figures $2 \mathrm{c}$ and $2 \mathrm{~d}$ show the comparisons of the data with the predictions of Eqs. (2). The theoretical shear speed follows the data points satisfactorily and, at depths below $10 \mathrm{~cm}$, the theoretical intrinsic attenuation curve coincides with the lower bound of the effective attenuation data. At shallower depths, Richardson [16] has suggested that the data are under-estimated due to ducting in the steep sound speed profile.

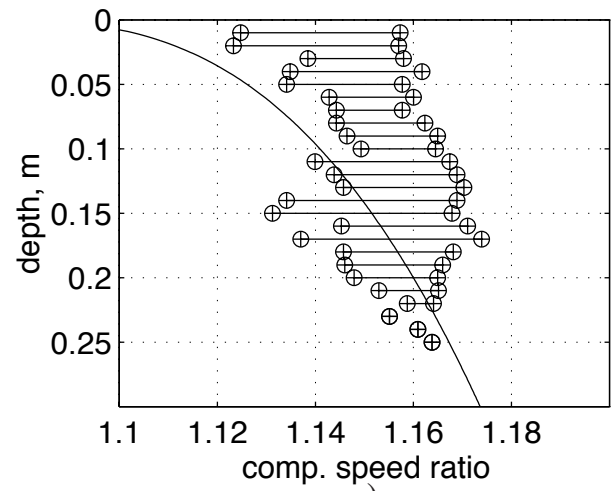

a)

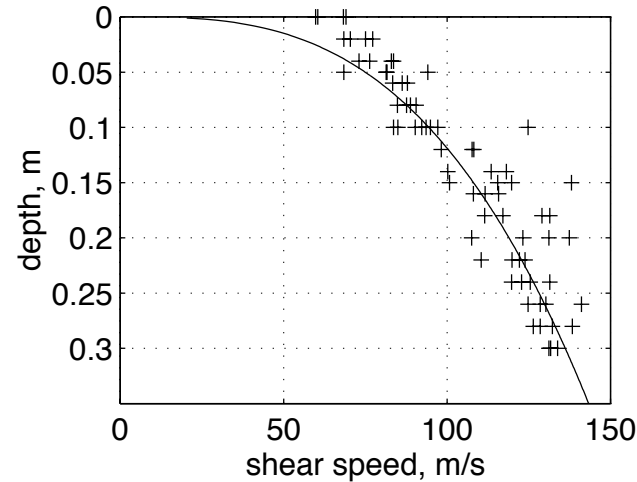

c)

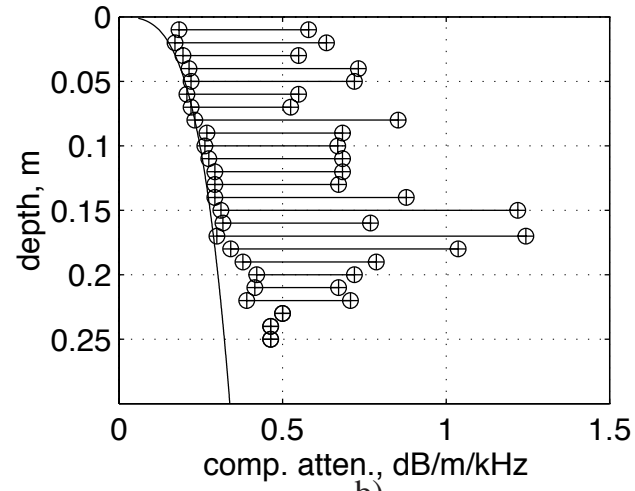

b)

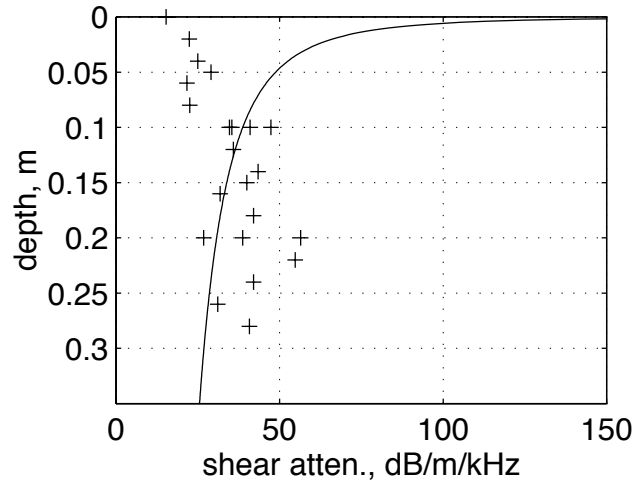

d)

FIGURE 2. Depth dependence of a) the sound speed ratio, b) the sound attenuation, c) the shear speed, and d) the shear attenuation.

\section{POROSITY DEPENDENCE}

Richardson and colleagues have made numerous measurements, on core samples and in situ, of the phase speeds and attenuations of compressional waves and shear waves in a wide variety of siliciclastic sediments. The materials range from coarse sands to clays, spanning porosities from 0.37 to 0.9 . Their core data on compressional wave properties are summarized in [5]. Figures $3 \mathrm{a}$ and $3 \mathrm{~b}$ show mostly in situ data for the compressional wave speed (normalized to the sound speed in seawater) and attenuation versus porosity. 


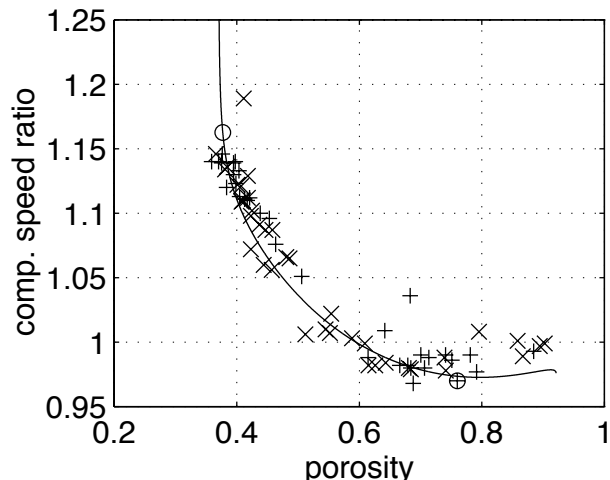

a)

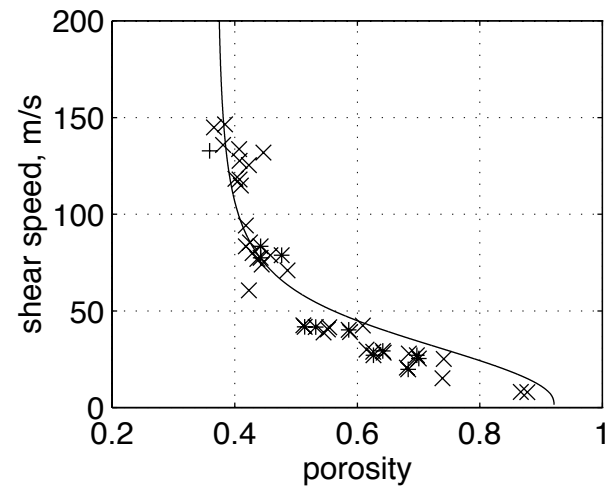

c)

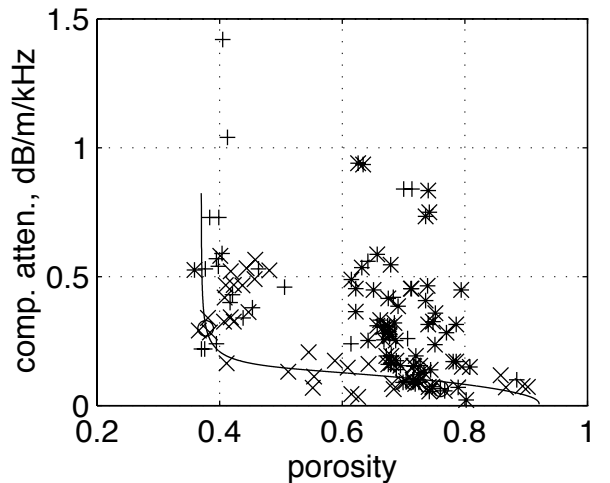

b)

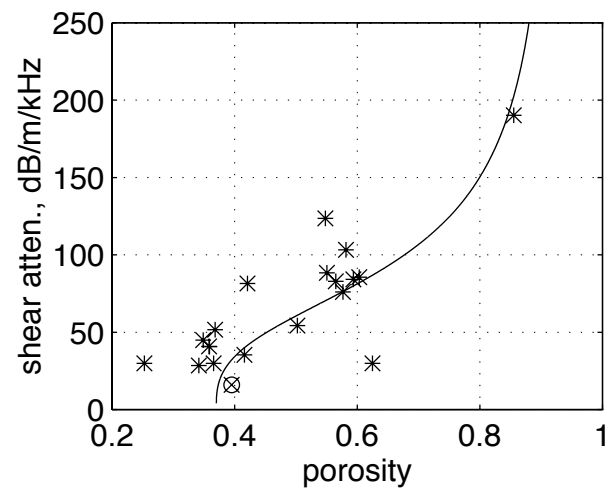

d)

FIGURE 3. Porosity dependence of a) the sound speed ratio, b) the sound attenuation, c) the shear speed, and d) the shear attenuation.

In Fig. 3a, the theoretical dispersion curve accurately follows the trend of the data points throughout the full porosity range. Note the extremely high gradient of both the curve and the data in the low porosity region, $N<0.4$. The broad theoretical minimum in the vicinity of $N=0.8$ is primarily due to a similar minimum in the Wood's sound speed, $c_{o}$, appearing in the numerator of Eq. (1a).

The effective attenuation data in Fig. $3 b$ are widely scattered, falling mostly above the theoretical curve, which represents the intrinsic attenuation. As in Fig. 2b, the theory traces out the lower bound of the attenuation data, as it should. Once again, a very high gradient appears in the low porosity region, where $N<0.4$.

Figure 3c shows Richardson's shear-wave speed and attenuation data as functions of porosity. The theoretical curve for the shear speed represents well the trend of the data, with both theory and measurements showing an extremely high gradient when $N<0.4$. Although fewer data on shear attenuation are available, Richardson [17] has reported a sufficient number of measurements to identify a trend, as can be seen in Fig. $3 \mathrm{~d}$. Consistent with the previous attenuation comparisons, the theoretical curve representing the intrinsic attenuation in Fig. $3 d$ delineates the lower bound of the effective attenuation data (apart from one rogue point at $N \approx 0.63$ ). 


\section{GRAIN SIZE DEPENDENCE}

Richardson's measurements of wave properties versus porosity usually include an estimate of the mean grain diameter, with the coarser materials corresponding to the lower porosities. As discussed by Buckingham [9], the compressional and shear wave dispersion relationships in Eqs. (1) and (2) match the grain-size data with a quality similar to that shown in the porosity plots of Fig. 3. Both the compressional and shear wave speed follow the trends of the grain-size data accurately; and both of the predicted intrinsic attenuations outline the lower bounds of the widely scattered, effective attenuation data points.

\section{CONCLUDING REMARKS}

Two theoretical dispersion pairs, one for the compressional wave and the second for the shear wave in a marine sediment, have been compared with extensive data sets on the wave properties of sediments. The theory, which indicates that the compressional and shear wave properties are coupled, has no adjustable parameters. All the comparisons of the theory with data - wave speeds and attenuations versus frequency, depth in the sediment, porosity and grain size - show a high level of agreement. It has been demonstrated that the theoretical curves accurately follow the trends of the data in every case. This agreement extends even to the attenuation: the theory, which predicts intrinsic attenuation, due to the conversion of wave energy into heat, traces out the lower bound to the highly scattered attenuation data points. The latter represent the effective attenuation, which includes additional loss mechanisms such as scattering from shell fragments and other inhomogeneities in the medium.

The precision of the theory of wave propagation in marine sediments suggests that it has practical application in characterizing the geoacoustic properties of the seabed. All that is needed is a single measurement of either a wave property, say the compressional wave speed, or a physical property such as the porosity from a core sample. The remaining geoacoustic parameters may then be computed from a few simple algebraic expressions, which include the two sets of dispersion relationships representing the wave properties of the compressional and the shear wave.

\section{ACKNOWLEDGMENTS}

This work was supported by Dr. Ellen Livingston, Ocean Acoustics Code, the Office of Naval Research, under grant number N00014-04-1-0063.

\section{REFERENCES}

1. M. A. Biot, J. Acoust. Soc. Am. 28, 168-178 (1956).

2. M. A. Biot, J. Acoust. Soc. Am. 28, 179-191 (1956).

3. E. L. Hamilton and R. T. Bachman, J. Acoust. Soc. Am. 72, 1891-1904 (1982).

4. E. L. Hamilton, "Acoustic properties of sediments", in Acoustics and the Ocean Bottom, edited by A. Lara-Saenz, C. Ranz Cuierra, and C. Carbo-Fité, Consejo Superior de Investigacions Cientificas, Madrid, 1987, pp. 3-58. 
5. M. D. Richardson and K. B. Briggs, "Empirical predictions of seafloor properties based on remotely measured sediment impedance". in High Frequency Ocean Acoustics, edited by M. Porter, M. Siderius, and W. A. Kuperman, (2004).

6. M. J. Buckingham, J. Acoust. Soc. Am. 108, 2796-2815, (2000).

7. A. B. Wood, A Textbook of Sound, Bell and Sons, London, 1964.

8. S. P. Timoshenko and J. N. Goodier, Theory of Elasticity, McGraw-Hill, New York, 1970.

9. M. J. Buckingham, J. Acoust. Soc. Am. in preparation (2004).

10. M. J. Buckingham, J. Acoust. Soc. Am. 102, 2579-2596 (1997).

11. H. J. Simpson and B. H. Houston, J. Acoust. Soc. Am. 107, 2329-2337 (2000).

12. H. J. Simpson, et al., J. Acoust. Soc. Am. 114, 1281-1290 (2003).

13. M. J. Buckingham and M. D. Richardson, IEEE J. Ocean. Eng. 27, 429-453 (2002).

14. B. A. Brunson and R. K. Johnson, J. Acoust. Soc. Am. 68, 1371-1375 (1980).

15. M. D. Richardson, et al., IEEE J. Ocean. Eng. 26, 26-53 (2001).

16. Michael D. Richardson, private communication.

17. M. D. Richardson, "Variability of shear wave speed and attenuation in surficial marine sediments," in Impact of Littoral Environmental Variability on Acoustic Predictions and Sonar Performance, edited by N. G. Pace and F. B. Jensen, Kluwer, Dordrecht, 2002, pp. 107-114. 